\title{
PHOTOGRAMMETRIC PROCESSING OF OSIRIS-REX IMAGES OF ASTEROID (101955) BENNU
}

\author{
K. L. Edmundson ${ }^{1}$, , K. J. Becker ${ }^{1}$, T. L. Becker ${ }^{1}$, C. A. Bennett ${ }^{1}$, D. N. DellaGiustina ${ }^{1}$, D. R. Golish ${ }^{1}$, N. A. Porter ${ }^{1}$, B. Rizk ${ }^{1}$, C. \\ Drouet d'Aubigny ${ }^{1}$, M.G. Daly ${ }^{2}$, E. Palmer ${ }^{3}$, J. Weirich ${ }^{3}$, O.S. Barnouin ${ }^{4}$, L. Philpott ${ }^{5}$, M. M. Al Asad ${ }^{5}$, J. A. Seabrook ${ }^{2}$, C. L. \\ Johnson $^{5}$, D. S. Lauretta ${ }^{1}$ \\ ${ }^{1}$ Lunar and Planetary Laboratory, University of Arizona, Tucson, Arizona, USA - kedmunds@orex.lpl.arizona.edu \\ ${ }^{2}$ The Centre for Research in Earth and Space Science, York University, Toronto, Ontario, Canada - dalym@yorku.ca \\ ${ }^{3}$ Planetary Science Institute, Tucson, Arizona, USA - epalmer@psi.edu \\ ${ }^{4}$ The Johns Hopkins University Applied Physics Laboratory, Laurel, Maryland, USA - olivier.barnouin@jhuapl.edu \\ ${ }^{5}$ Department of Earth, Ocean \& Atmospheric Sciences, University of British Columbia, Vancouver, Canada - malasad@eoas.ubc.ca
}

\section{Commission III}

KEY WORDS: OSIRIS-REx, Mapping, OCAMS, Bennu, Asteroid, Photogrammetry

\begin{abstract}
:
The principal objective of the Origins, Spectral Interpretation, Resource Identification, and Security-Regolith Explorer (OSIRIS-REx) mission is to retrieve a sample of the asteroid (101955) Bennu and return it to Earth. OSIRIS-REx arrived at Bennu in December 2018. Images of the asteroid by the OSIRIS-REx Camera Suite (OCAMS) were photogrammetrically controlled to produce a global basemap and site-specific image mosaics essential to the selection of a primary and backup sample site, which were announced in December 2019. In the control process, OCAMS images were registered to shape models created from OSIRIS-REx Laser Altimeter (OLA) data and from the process of stereophotoclinometry. This paper summarizes the photogrammetric control to date of images collected at Bennu. We briefly review the mission and the OCAMS imaging sensors. We then describe the photogrammetric control process for the global mapping campaign and targeted reconnaissance surveys of candidate sample sites. Finally, we discuss ongoing and future work.
\end{abstract}

\section{INTRODUCTION}

Following a journey of more than 2 years, NASA's Origins, Spectral Interpretation, Resource Identification, and SecurityRegolith Explorer (OSIRIS-REx) spacecraft arrived at the nearEarth asteroid (101955) Bennu in December of 2018. Since then, the spacecraft and OSIRIS-REx team have been mapping Bennu and characterizing its geological, chemical, and physical properties in support of the primary mission objective to acquire a sample for return to Earth.

Characterized as a 'rubble-pile' asteroid (Barnouin et al., 2019; DellaGiustina et al., 2019), Bennu has a generally spheroidal shape resembling a spinning top (Figure 1). Equatorial radii are $282.37 \mathrm{~m} \mathrm{x} 268.05 \mathrm{~m}$ and the polar radius is $249.25 \mathrm{~m}$. Bennu's

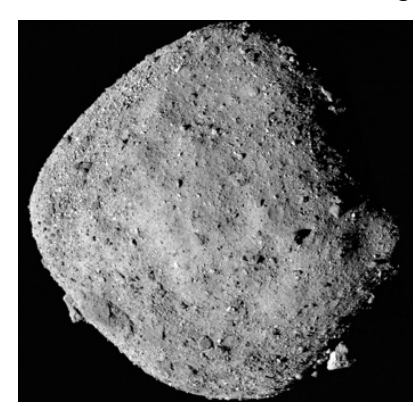

Figure 1. Mosaic of Bennu composed of 12 PolyCam images acquired December 2, 2018. Credit: NASA / Goddard / University of Arizona.

* Corresponding author environment, to a rough surface dominated by potential hazards such as craters, ridges, and large boulders (Lauretta et al., 2019). Success depends upon having accurately characterized the surface beforehand. To this end, OSIRIS-REx Camera Suite (OCAMS; Rizk et al., 2018) images acquired within the Detailed Survey and Reconnaissance portions of the mission (Figure 2) have been photogrammetrically controlled to generate panchromatic and color image basemaps at global and local scales. Control was accomplished with a modified version of the Integrated Software for Imagers and Spectrometers (ISIS) planetary cartography package developed and maintained by the OSIRIS-REx team (DellaGiustina et al., 2018; Sides et al., 2017).

These image products have informed the process of selecting a sample site with regard to science value, the availability of sampleable material (particles smaller than $2 \mathrm{~cm}$ that can be

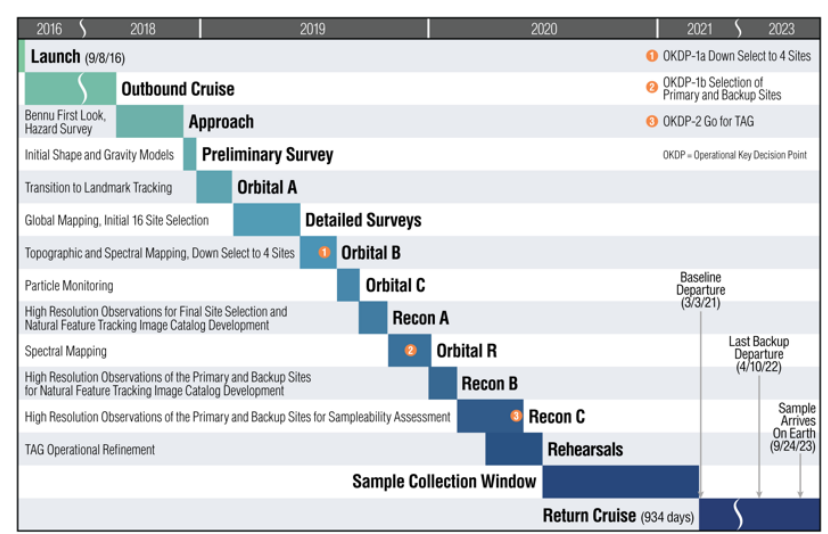

Figure 2. Timeline of OSIRIS-REx mission phases and associated science products (adapted from Lauretta et al., 2017). 
ingested by the OSIRIS-REx sampling mechanism; Bierhaus et al., 2018), and the presence of surface features that might prevent the spacecraft from approaching safely and retrieving a sample. They have enabled the iterative reduction of approximately 50 original candidate sites to $32,16,8,4$, and finally 2 - a primary and a backup. The primary and backup sample sites, designated Nightingale and Osprey, respectively, were announced in December 2019.

In the following we describe the OCAMS imaging sensors and associated lens distortion models; the photogrammetric control of OCAMS data acquired in the Detailed Survey and Reconnaissance A phases of the OSIRIS-REx mission; and summarize ongoing and future work.

\section{OCAMS: THE OSIRIS-REX CAMERA SUITE}

OCAMS (Figure 3; Table 1), consists of three 1-megapixel imaging sensors (SamCam, PolyCam, and MapCam) that perform a variety of functions throughout the mission. The wideangle SamCam records and validates the sampling procedure, whereas image-based mapping of Bennu is accomplished primarily with MapCam and PolyCam. PolyCam is a narrowangle, high-resolution, panchromatic sensor. With variable focus (94 focus positions), it can image Bennu both as a point source from a distance of several million kilometers and at subcentimeter resolution from a distance of $\sim 200 \mathrm{~m}$. The medium angle MapCam sensor acquires panchromatic data with a broadband filter and color data with four narrow-band filters. With a field of view five times that of PolyCam, MapCam images serve as context for PolyCam images acquired within the same observation.

Golish et al. (2020a) describe OCAMS pre- and in-flight calibration procedures. Ground calibrations were conducted for all three OCAMS cameras to characterize bias level, dark current rates, flat field response, stray light, and radiometric performance. MapCam interior orientation including lens distortion was determined for each filter with star field images acquired in flight. PolyCam distortion was established pre-flight at $200 \mathrm{~m}$ and with star field images for longer ranges. SamCam lens distortion is yet to be determined.

\subsection{MapCam Distortion Model}

The MapCam distortion model (Pelgrift et al., 2018) includes radial symmetric distortion that displaces image points radially from the principal point and decentering distortion (Brown, 1966) which is caused by errors in the alignment of lens components and produces both a radial and tangential displacement. An additional parameter models the temperature dependence of the focal length. Temperature dependence was determined by performing star calibrations at a range of temperatures that were anticipated during operation.

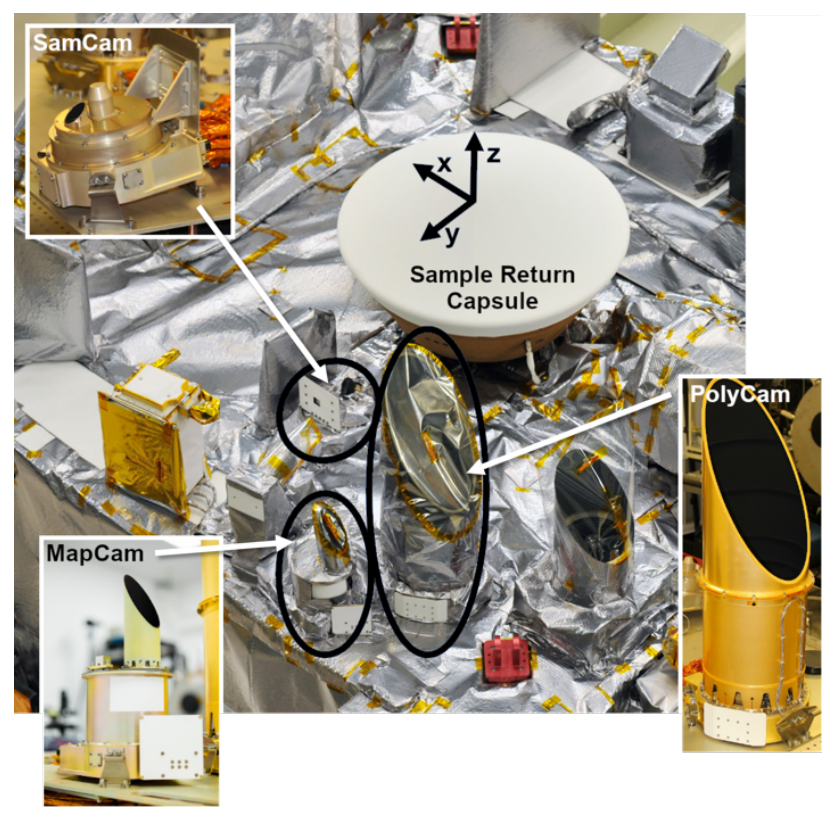

Figure 3. OCAMS mounted to the OSIRIS-REx science deck during thermal vacuum testing (after Rizk et al., 2018). Axes indicate the spacecraft coordinate system. The XY plane lies flush with the science deck. Credits: NASA/Goddard/Lockheed Martin/University of Arizona.

Radial distortion is given by

$\delta r=k_{1} r^{3}+k_{2} r^{5}+k_{3} r^{7}$

where $\delta r$ is the total radial displacement, the $k_{i}$ are coefficients of radial distortion, and $r$ is the radial distance from the principal point such that

$r^{2}=\bar{x}^{2}+\bar{y}^{2}$

where $(\bar{x}, \bar{y})$ are the coordinates of an undistorted image point reduced to the principal point.

Resolution of the total radial displacement into its $x$ and $y$ components yields

$\delta x_{r}=\bar{x} \delta r / r$

$\delta y_{r}=\bar{y} \delta r / r$

The $x$ and $y$ components of decentering distortion are given by

$\delta x_{d}=P_{1}\left(r^{2}+2 \bar{x}^{2}\right)+2 P_{2} \bar{x} \bar{y}$

$\delta y_{d}=2 P_{1} \bar{x} \bar{y}+P_{2}\left(r^{2}+2 \bar{y}^{2}\right)$

where the $P_{i}$ are the coefficients of decentering distortion.

Table 1. OCAMS sensor characteristics. PolyCam focal lengths are given at either end of the focal range. SamCam lens distortion is yet to be determined.

\begin{tabular}{|r|c|c|c|c|}
\hline & SamCam & \multirow{2}{*}{ MapCam } & \multicolumn{2}{|c|}{ PolyCam } \\
\cline { 3 - 4 } & & & $200 \mathrm{~m}$ & $\infty$ \\
\hline focal length $(\mathrm{mm})$ & 24 & 125 & 610.2 & 628.9 \\
\hline CCD format & $1024 \times 1024$ & $1024 \times 1024$ & $1024 \times 1024$ \\
\hline pixel size $(\mu \mathrm{m})$ & 8.5 & 8.5 & 8.5 \\
\hline field of view (degrees) & $20.8 \times 20.8$ & $4.0 \times 4.0$ & $0.8 \times 0.8$ \\
\hline lens aperture & $\mathrm{f} / 5.6$ & $\mathrm{f} / 3.3$ & $\mathrm{f} / 3.5$ \\
\hline maximum distortion & N/A & $13.6 \mu \mathrm{m}$ & $18.7 \mu \mathrm{m}$ \\
\hline
\end{tabular}


Distorted image coordinates $\left(x^{\prime}, y^{\prime}\right)$ are then

$x^{\prime}=\bar{x}+\delta x_{r}+\delta x_{d}$

$y^{\prime}=\bar{y}+\delta y_{r}+\delta y_{d}$

Distorted image coordinates are converted to sample and line pixels $(u, v)$ with

$u=f_{x}\left(1+a_{1} T\right) x^{\prime}+c_{x}$

$v=f_{y}\left(1+a_{1} T\right) y^{\prime}+c_{y}$

where $a_{1}$ models the temperature dependency of the focal length, $f_{x}, f_{y}$ represent the focal length in pixels at $0^{\circ} \mathrm{C}$ in the image plane $x$ and $y$ directions, $c_{x}, c_{y}$ are the pixel coordinates of the principal point, and $T$ is the temperature in degrees Celsius.

\subsection{PolyCam Distortion Model}

Principal point and radial distortion parameters in the PolyCam model vary as a function of focus position. Modelled differently from MapCam (Golish et al., 2020a), radial distortion is given as

$\delta r=\rho_{1} r+\rho_{2} r^{2}+\rho_{3} r^{3}$

Here again, $\delta r$ is the total radial displacement and $r$ is the radial distance from the principal point. Radial distortion coefficients are given as $\rho_{i}$ to distinguish them from the MapCam radial distortion model. PolyCam focal length, principal point, and radial distortion parameters were established at 94 focus positions. Focal length and the $\rho_{1}$ coefficient are completely correlated. To enable solving for focal length, the $\rho_{1}$ coefficient was fixed to 0.0 in the PolyCam calibrations.

\section{IMAGE PLANNING AND PRE-PROCESSING}

Science observation planning for OSIRIS-REx (Polit et al., 2020) is accomplished primarily with the J-Asteroid geospatial information system developed by the Arizona State University Mars Space Flight Facility (Christensen et al., 2018; Figure 4). Planning for image acquisition considers, for example, pixel and footprint size; emission, phase, and incidence angles; local solar hour; target coverage; and image overlap.

Prior to photogrammetric control, images are pre-processed (Golish et al., 2020a) to remove bias, dark counts, bad pixels, and charge smear. Flat-field correction is applied and images are converted into units of reflectance (I/F). Images are then converted into ISIS cube format and additional pre-processing is performed with ISIS.

\section{THE GLOBAL MAPPING CAMPAIGN}

PolyCam, MapCam, and OSIRIS-REx Laser Altimeter (OLA; Daly et al., 2017) observations were acquired over seven

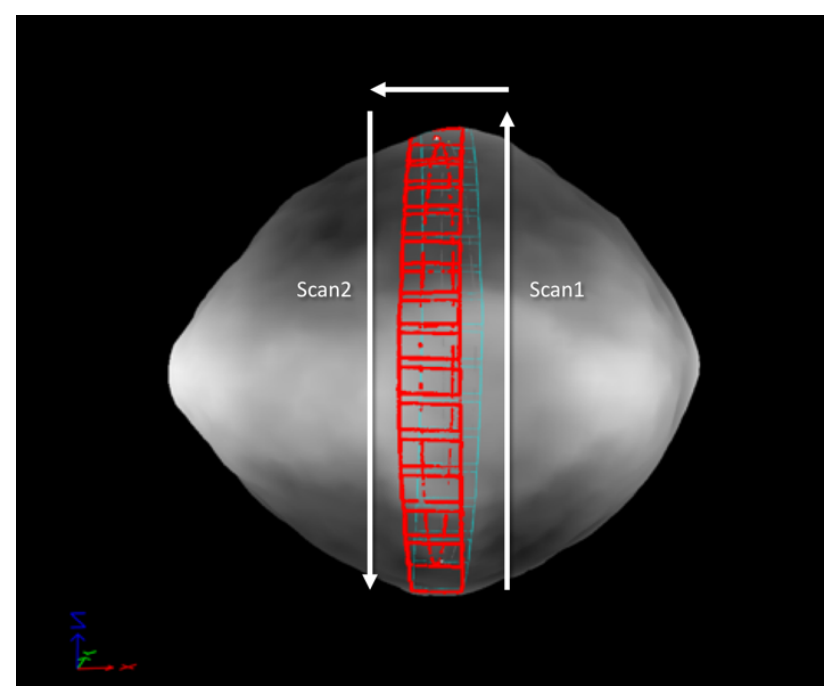

Figure 4: Example of science observation planning for the global panchromatic basemap with the J-Asteroid geospatial information system (Christensen et al., 2018). Shown are the first two image scans for Flyby 3 in the Detailed Survey: Baseball Diamond subphase of the mission. Scan 1 (in blue) was acquired as the spacecraft slewed from south to north. Scan 2 (in red) follows immediately in the opposite direction. Ninety-two scans were acquired for Flyby 3 to achieve global coverage.

hyperbolic flybys $(1,2,3,4 \mathrm{~A} / \mathrm{B}, 5 \mathrm{~A} / \mathrm{B}, 6 \mathrm{~A} / \mathrm{B}, 7 \mathrm{~A} / \mathrm{B})$ of Bennu during March and April of 2019 in the Detailed Survey: Baseball Diamond subphase. In the microgravity environment of Bennu, optimal spacecraft stability can be attained only in a terminator orbit owing to the influence of solar radiation pressure. However, image quality is highly variable from a terminator orbit. Nadirlooking images in the vicinity of the terminator suffer from poor illumination. Oblique images may be better illuminated but will exhibit foreshortening, reduced resolution, and poor focus for terrain beyond the camera's depth of field. Hyperbolic flybys offer better imaging geometry (DellaGiustina et al., 2018). Below, following closely Bennett et al. (2020), we summarize the photogrammetric control of OCAMs PolyCam images to produce a global panchromatic basemap of Bennu. Efforts to control MapCam color images are ongoing.

\section{GLOBAL PANCHROMATIC BASEMAP}

\subsection{Image Acquisition}

PolyCam images controlled for the panchromatic basemap were obtained primarily from Flybys 3, 4A, and 4B. In each flyby, the spacecraft was $\sim 3.5 \mathrm{~km}$ from Bennu and maintained a constant sub-spacecraft latitude. As the spacecraft passed over the surface, it slewed alternately north and south acquiring images in a serpentine fashion. The average pixel scale was $\sim 5 \mathrm{~cm}$ and image overlaps ranged from $38 \%$ to $49 \%$ in the north-south direction and from $30 \%$ to $50 \%$ east-west (Figure 4; Table 2). A small

Table 2. Summary of imaging flybys for the global panchromatic basemap (after Bennett et al., 2020).

\begin{tabular}{|r|c|c|c|c|c|c|c|c|}
\hline \multirow{2}{*}{ Flyby } & \multirow{2}{*}{$\begin{array}{c}\text { Images acquired } / \\
\text { used in basemap }\end{array}$} & \multirow{2}{*}{$\begin{array}{c}\text { Sub-spacecraft } \\
\text { latitude }\end{array}$} & \multirow{2}{*}{ Coverage } & \multicolumn{2}{|c|}{ Mean photometric angles } & \multicolumn{2}{c|}{$\%$ Image overlap } \\
\cline { 5 - 9 } & & & Phase & Emission & Incidence & $\mathrm{N} / \mathrm{S}$ & $\mathrm{E} / \mathrm{W}$ \\
\hline 3 & $1365 / 641$ & $0^{\circ}$ & $70^{\circ} \mathrm{S}-70^{\circ} \mathrm{N}$ & $30.46^{\circ}$ & $14.70^{\circ}$ & $34.03^{\circ}$ & $38-44$ & $30-40$ \\
\hline 4A & $976 / 754$ & $40^{\circ} \mathrm{S}$ & $90^{\circ} \mathrm{S}-0^{\circ}$ & $48.66^{\circ}$ & $23.18^{\circ}$ & $55.34^{\circ}$ & $38-49$ & $30-50$ \\
\hline 4B & $1037 / 751$ & $40^{\circ} \mathrm{N}$ & $0^{\circ}-90^{\circ} \mathrm{N}$ & $48.46^{\circ}$ & $22.62^{\circ}$ & $52.07^{\circ}$ & $41-45$ & $30-50$ \\
\hline $5 \mathrm{~A}$ & $837 / 3$ & $40^{\circ} \mathrm{N}$ & $0^{\circ}-90^{\circ} \mathrm{N}$ & $39.45^{\circ}$ & $29.93^{\circ}$ & $64.54^{\circ}$ & $30-50$ & $20-40$ \\
\hline 6A & $837 / 6$ & $40^{\circ} \mathrm{S}$ & $90^{\circ} \mathrm{S}-0^{\circ}$ & $39.60^{\circ}$ & $28.48^{\circ}$ & $65.19^{\circ}$ & $30-50$ & $20-40$ \\
\hline
\end{tabular}


number of images from Flybys 5A and 6A were included to cover regions at high latitudes insufficiently illuminated in Flybys 3, $4 \mathrm{~A}$, and $4 \mathrm{~B}$.

\subsection{Image Measurement}

Tie point image measurements are acquired in ISIS by automatic interest point detection and image matching with the findfeatures application. The pointreg application was then used to refine these measurements to sub-pixel accuracy (Becker et al., 2017). Although outlier rejection in the matching process was highly successful, some manual editing was required to correct mismatches occurring between images with notably different geometry and illumination.

Ground control points (GCPs) were obtained from 32 local digital terrain models (LDTMs) corresponding to candidate sample sites and the location of the prime meridian. These were generated from OLA data at a resolution of $\sim 15 \mathrm{~cm}$. The LDTMs are registered to a global DTM (GDTM) generated via stereophotoclinometry at a resolution of $\sim 80 \mathrm{~cm}$ (Barnouin et al., 2019, 2020; Gaskell et al., 2008). The accuracy of the GDTM was estimated to be $0.52 \mathrm{~m}$ horizontally and $0.64 \mathrm{~m}$ radially. The LDTMs were utilized for GCP measurement as they were created at resolutions closer to that of the OCAMS images. GCPs were measured manually from terrain-shaded relief (TSR) maps that were created for each LDTM to facilitate visual correlation between surface features in the images and the LDTM. Because the LDTMs were only available at candidate sample sites and were not uniformly distributed over Bennu, the resulting GCP distribution was less than optimal, particularly in the southern hemisphere.

\subsection{Bundle Adjustment}

The final control network consisted of 3142 images, 792,217 tie points, 127 GCPs, and 3,257,452 image measurements. Bundle adjustments were performed with the ISIS program jigsaw (Edmundson et al., 2012). Initial image exterior orientation parameters were obtained from reconstructed NAIF spacecraft position and camera pointing kernels (Acton, 1996). Initial coordinates for tie points were determined by first projecting all image measurements through the sensor model to obtain the intersection with the GDTM. For each tie point, all measurement intersection points were averaged to obtain coordinates for input to the bundle adjustment.

After adjustment, the root mean square (rms) of all image measurement residuals was $\sim 0.082$ pixels. The rms of image measure residuals for GCPs only was $\sim 1.0$ pixels, equating to $\sim 5$ $\mathrm{cm}$ or one-third of the average resolution of the LDTMs. To estimate the spatial accuracy of the solution, we performed a separate bundle adjustment in which 11 GCPs were constrained and the remaining 116 were treated as checkpoints. The rms of checkpoint coordinate discrepancies was $23.3,19.2$, and $30.7 \mathrm{~cm}$ in latitude, longitude and radius respectively. To assess the fit between the adjusted point cloud and the GDTM, we compared the adjusted radius coordinate of each point to the radius at the corresponding location in the GDTM. The mean absolute error of these differences was $\sim 47.0 \mathrm{~cm}$. Approximately $90 \%$ of points lie within $1 \mathrm{~m}$ of the GDTM. The rest deviate from the surface as much as $\sim 22.0 \mathrm{~m}$ with the highest differences around large boulders and on crater floors (Bennett et al., 2020).

\subsection{Creating the Basemap}

The final basemap (Figures 5, 6) was produced with 2155 of the 3142 images in the original control network. Separate mosaics were first created for each of Flybys 3, 4A, and 4B. For each flyby mosaic, images were added in the order of acquisition, thus the more recent images were on top. The flyby mosaics were then combined to produce the global basemap. Where the flybys overlap, precedence was generally given to regions with the lowest overall emission angle while at the same time attempting to minimize abrupt seams. Images from Flybys $5 \mathrm{~A}$ and $6 \mathrm{~A}$ were utilized to fill areas of Flybys $4 \mathrm{~A}$ and $4 \mathrm{~B}$ that were completely shadowed. The basemap was photometrically normalized using a Minnaert phase angle correction (Golish et al., 2020b)

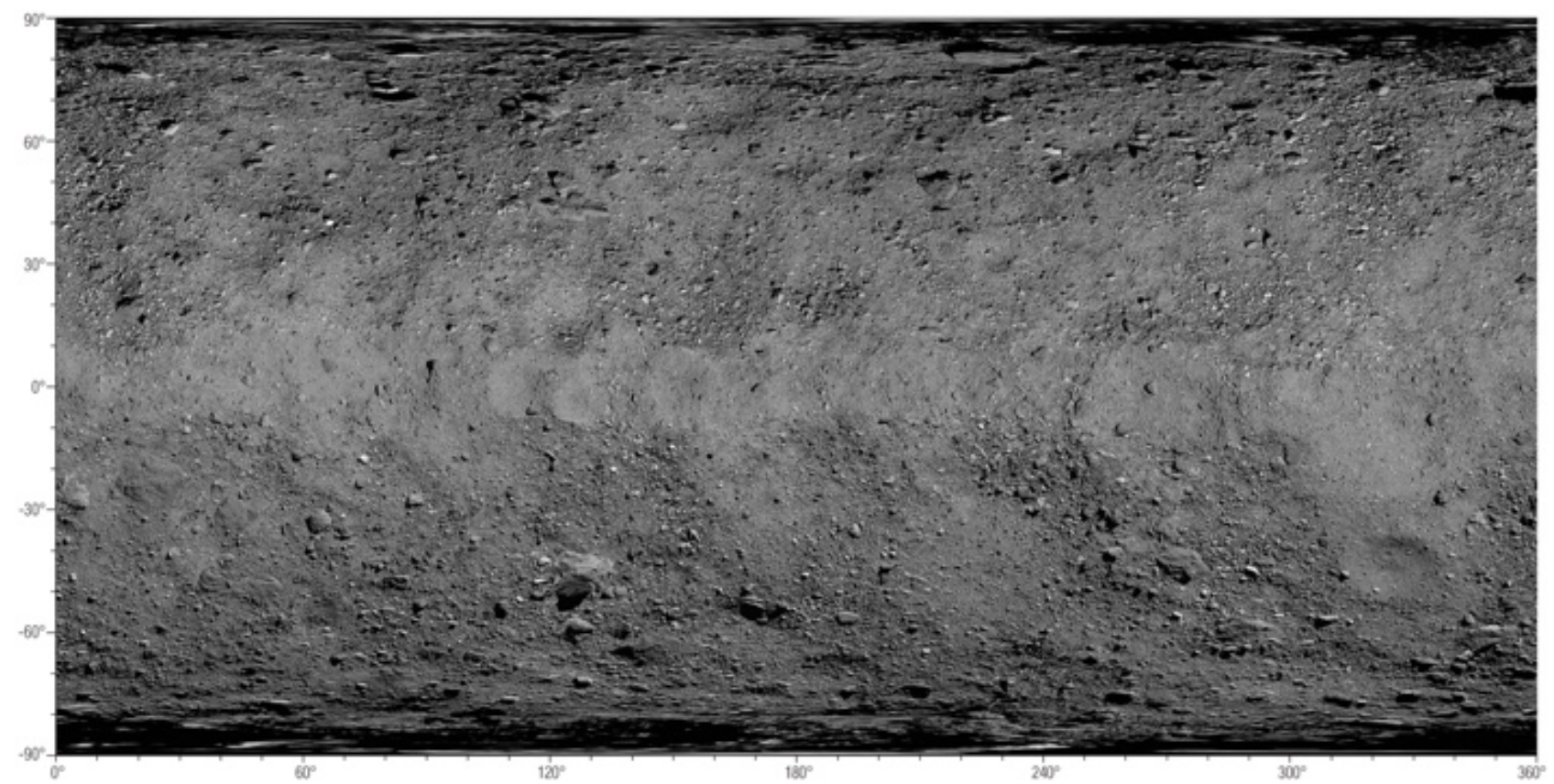

Figure 5. Global panchromatic basemap of Bennu created from 2155 OCAMS PolyCam images acquired at a pixel scale of $\sim 5 \mathrm{~cm}$ (Bennett et al., 2020). Projection is equirectangular. 

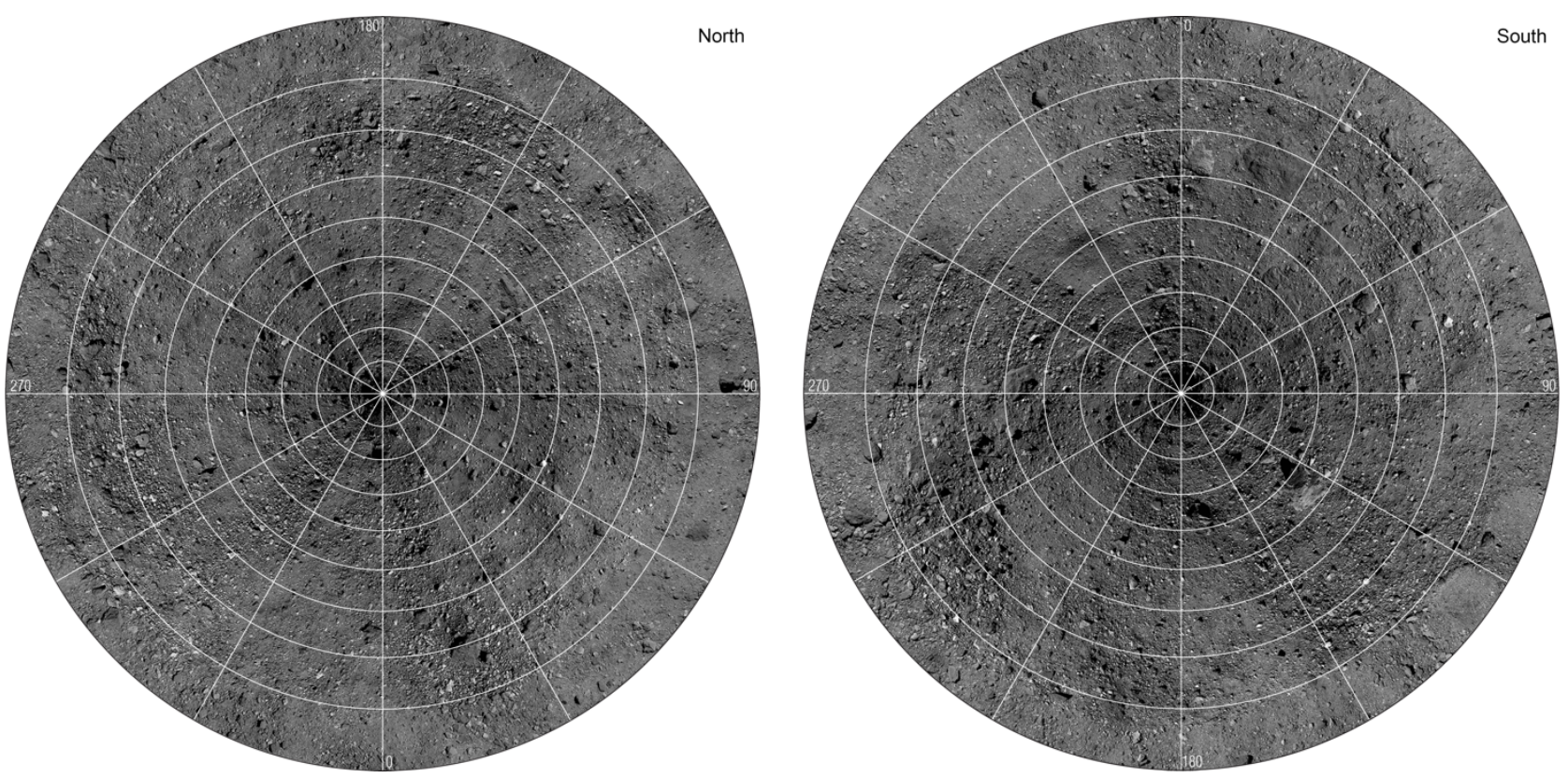

Figure 6. Global basemap north and south views in polar stereographic projection (Bennett et al., 2020). Latitude ranges from $0^{\circ}$ to $90^{\circ} \mathrm{N}$ (left) and $0^{\circ}$ to $90^{\circ} \mathrm{S}$ (right). Latitude grid is in $10^{\circ}$ increments, starting from $0^{\circ}$ at the outermost mark.

\section{SURVEYS OF CANDIDATE SAMPLE SITES}

The final four candidate sample sites (Nightingale, Osprey, Kingfisher, and Sandpiper) were announced in August 2019. In October, these were further evaluated for safety and sampleability during the Reconnaissance A phase of the mission (Becker et al., 2020). Approximately 50 PolyCam images were acquired for each site and surrounding region with an average pixel scale of $\sim 1.4 \mathrm{~cm}$ (Table 3 ). In each case the spacecraft was $\sim 1.015 \mathrm{~km}$ above the site. Image acquisition was accomplished in a manner similar to that for the global basemap. The linear camera station geometry for the Sandpiper site shown in Figure 7 was typical for all four sites. A small number of images per site

Table 3: Locations and approximate image overlaps for the final four candidate sample sites (single and double asterisks indicate the selected primary and secondary sample sites respectively).

\begin{tabular}{|c|c|c|c|}
\hline \multirow{2}{*}{ site } & \multirow{2}{*}{ location } & \multicolumn{2}{|c|}{ image overlap \% } \\
\cline { 3 - 4 } & $\mathrm{N} / \mathrm{S}$ & $\mathrm{E} / \mathrm{W}$ \\
\hline${ }^{*}$ Nightingale & $56^{\circ} \mathrm{N}, 43^{\circ} \mathrm{E}$ & 30 & $10-30$ \\
\hline${ }^{* *}$ Osprey & $11^{\circ} \mathrm{N}, 88^{\circ} \mathrm{E}$ & $33-44$ & 10 \\
\hline Kingfisher & $11^{\circ} \mathrm{N}, 56^{\circ} \mathrm{E}$ & $33-51$ & $20-25$ \\
\hline Sandpiper & $47^{\circ} \mathrm{S}, 322^{\circ} \mathrm{E}$ & $35-40$ & $5-10$ \\
\hline
\end{tabular}

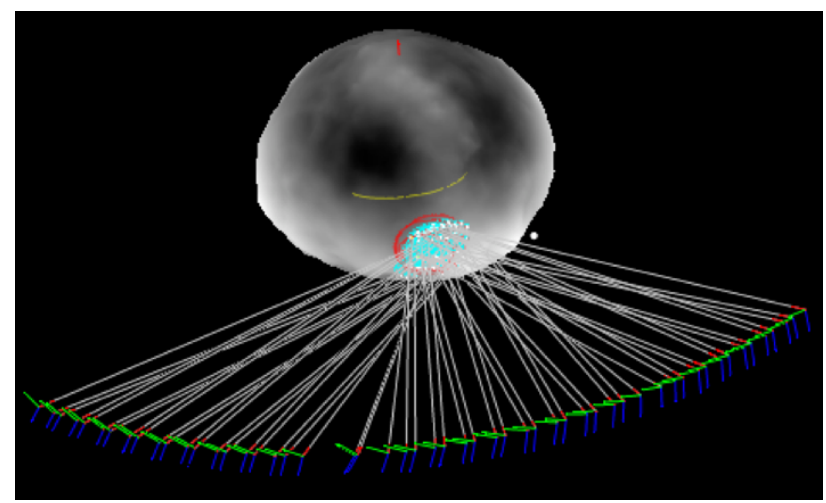

Figure 7. Spacecraft locations for Reconnaissance A image acquisition of site Sandpiper, displayed in J-Asteroid. Note the lack of diversity in camera station geometry. (three to six) were quickly photogrammetrically controlled and mosaiced to assess the availability of sampleable material and the presence of potentially hazardous features.

Tie point image measurements were again made by automatic interest point detection and image matching followed by subpixel refinement. GCPs were obtained from LDTMs produced from OLA data at a resolution of $\sim 5 \mathrm{~cm}$. Images are registered to a GDTM with a resolution of $\sim 80 \mathrm{~cm}$.

An alternative to the use of TSR maps to facilitate GCP measurement was taken here. Utilizing the LDTM, the image exterior orientation, and a photometric model of the surface, a simulated image with backplanes containing spatial information was created for each PolyCam image (Figure 8; Golish et al., 2017; Becker et al., 2020). GCP measurements were then obtained by visual correlation between a PolyCam image and its corresponding simulated image. The TSR and simulated image approaches are similar and equally accurate in terms of feature location. However, it has proven to be easier to identify features in the simulated images as they are notably more realistic in appearance due to the accurate representation of the light source and the presence of shadows.

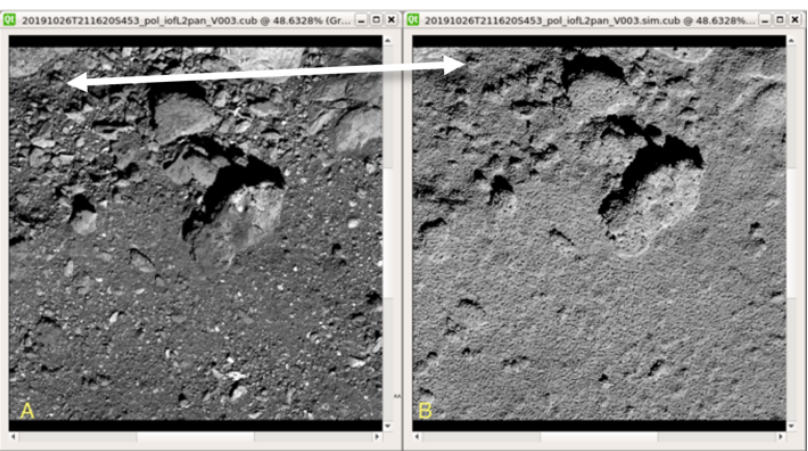

Figure 8. Left (A): input PolyCam I/F image. Right (B): image simulated with a priori exterior orientation, OLA DTM, and photometric model (from Becker et al., 2020). The doubleheaded arrow indicates a corresponding feature in both images. 
Bundle adjustment of these sites was complicated by the lack of diversity in camera station geometry (e.g. Figure 7) and less than optimal GCP distribution. High correlations between spacecraft position and pointing parameters for Nightingale, Osprey, and Kingfisher required suppression of one or the other in the solution to avoid ill-conditioning in the normal equations. We solved for pointing only for Nightingale; position only for Osprey and Kingfisher; and both position and pointing for Sandpiper.

Bundle adjustment statistics for the four sites are given in Table 4 and the resulting image mosaics with corresponding control networks are shown in Figure 9. Visual examination of the mosaics indicated that image-to-image registration is within one pixel. Checkpoint analyses gave accuracies in latitude and longitude of 2.1 and $5.7 \mathrm{~cm}$ for the Nightingale site; 4.3 and 4.5 $\mathrm{cm}$ for Osprey; and 11.5 and $10.5 \mathrm{~cm}$ for Kingfisher. As checkpoints were not available for the Sandpiper site, assessment was made by visual comparison between the updated images after adjustment and their corresponding simulated images. Here the accuracy was estimated to be $\sim 1.5 \mathrm{~cm}$ in latitude and longitude.

Table 4. Bundle adjustment statistics for final four candidate sample sites (single and double asterisks indicate the selected primary and secondary sample sites respectively).

\begin{tabular}{c|ccccc} 
& $\begin{array}{c}\# \\
\text { images }\end{array}$ & $\begin{array}{c}\# \\
\text { tie } \\
\text { points }\end{array}$ & $\begin{array}{c}\# \\
\text { control } \\
\text { points }\end{array}$ & $\begin{array}{c}\# \\
\text { image } \\
\text { measures }\end{array}$ & $\begin{array}{c}\text { rms } \\
\text { residuals } \\
\text { (pixels) }\end{array}$ \\
*Nightingale & 6 & 132 & 10 & 322 & 0.93 \\
** Osprey & 6 & 89 & 20 & 246 & 0.69 \\
Kingfisher & 4 & 44 & 11 & 119 & 0.92 \\
Sandpiper & 3 & 177 & 4 & 385 & 0.08 \\
\hline
\end{tabular}

\section{ONGOING AND FUTURE WORK}

OCAMS MapCam images also acquired in the Detailed Survey phase have been controlled to produce color global basemaps at $\sim 24 \mathrm{~cm} /$ pixel. These maps provide a multispectral description of Bennu's surface at spatial scales of $\sim 25 \mathrm{~cm} /$ pixel. Moving forward, we aim to globally control MapCam multispectral data to the higher-resolution PolyCam images in an effort to merge the color signatures on the asteroid with the more detailed surface morphology revealed by the monochrome PolyCam controlled mosaics.

Additionally, several localized site-specific observations have been acquired by both MapCam and PolyCam. Merging the sitespecific and globally controlled image networks is of high interest for the purposes of future geological mapping efforts and to gain context for the fine-scale processes that may influence larger-scale surface features on asteroid Bennu.

OCAMS images are currently available via the Planetary Data System (PDS; https://sbn.psi.edu/pds/resource/orex/). Higherlevel products (e.g. mosaics and shape models) will be available in the PDS one year after OSIRIS-REx departs Bennu.

\section{ACKNOWLEDGEMENTS}

This material is based upon work supported by NASA under Contract NNM10AA11C issued through the New Frontiers Program. We are grateful to the OSIRIS-REx Team.

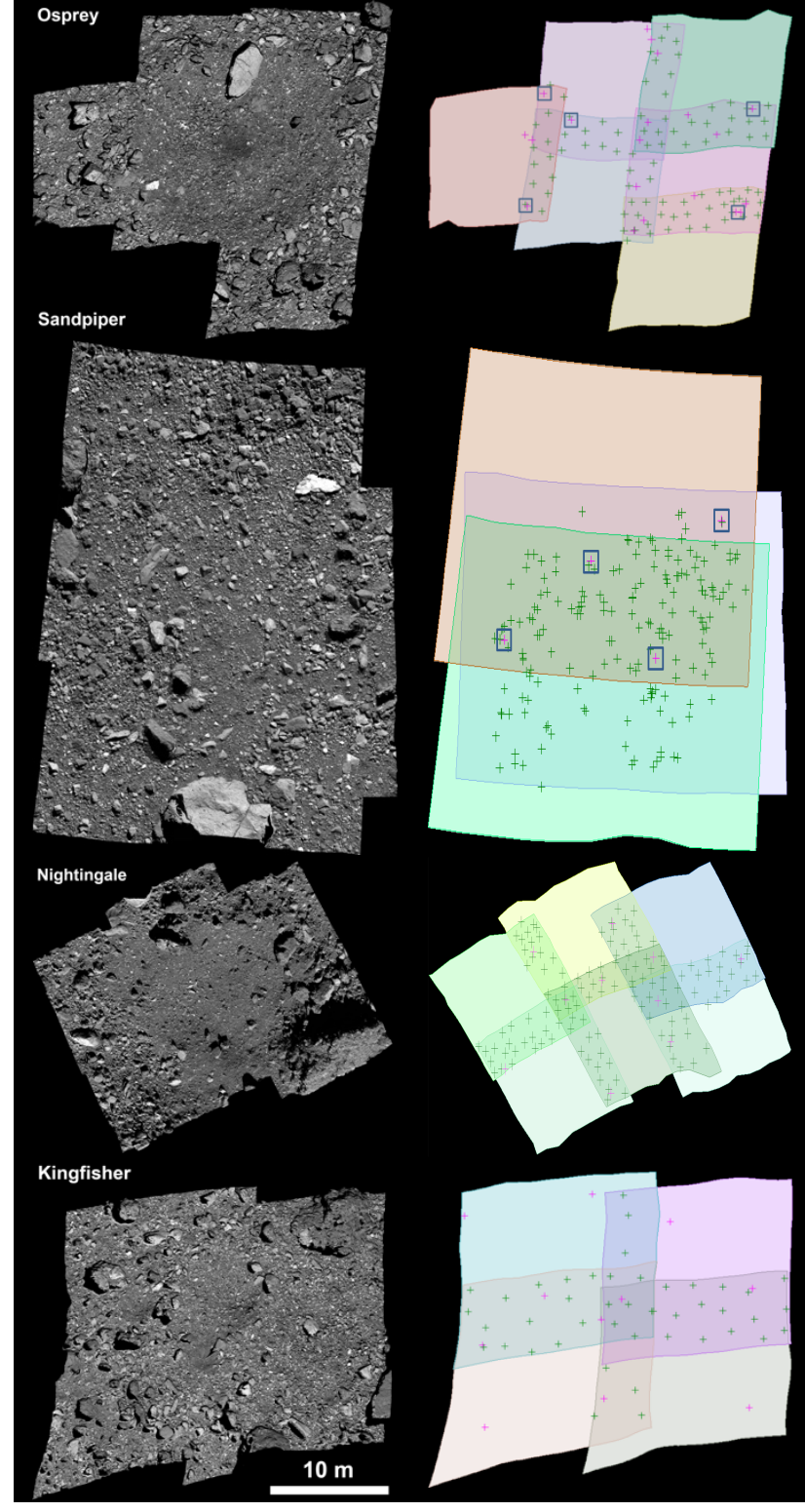

Figure 9. Left: controlled image mosaics of the final four candidate sample sites generated during Reconnaissance A and utilized in the evaluation of safety and sampleability. Right: image footprints corresponding to each mosaic, overlaid with control point networks.

\section{REFERENCES}

Acton, C.H., 1996. Ancillary Data Services of NASA's Navigation and Ancillary Information Facility. Planet. Space Sci., 44(1), 65-70, doi.org/10.1016/0032-0633(95)00107-7.

Barnouin, O.S., Nolan, M.C., 2019. Bennu Coordinate System Description. In: Crombie, M.K., and S. Selznick, Eds., Origins, Spectral Interpretation, Resource Identification, Security, Regolith Explorer (OSIRIS-REx): Mission Bundle, urn:nasa:pds:orex.mission, NASA Planetary Data System, https://sbnarchive.psi.edu/pds4/orex/orex.mission/document/Be nnu_Coordinate_System_Description.pdf, accessed 3 May 2020.

Barnouin, O.S., Daly, M.G., Palmer, E.E., Gaskell, R.W., Weirich, J.R., Johnson, C.L., Al Asad, M.M., Roberts, J.H., Perry, M.E., Susorney, H.C.M., Daly, R.T., Bierhaus, E.B., Seabrook, J.A., Espiritu, R.C., Nair, A.H., Nguyen, L., Neumann, 
G.A., Ernst, C.M., Boynton, W.V., Nolan, M.C., Adam, C.D., Moreau, M.C., Rizk, B., Drouet D’Aubigny, C.Y., Jawin, E.R., Walsh, K.J., Michel, P., Schwartz, S.R., Ballouz, R.-L., Mazarico, E.M., Scheeres, D.J., McMahon, J.W., Bottke, W.F., Sugita, S., Hirata, N., Hirata, N., Watanabe, S.-I, Burke, K.N., DellaGiustina, D.N., Bennett, C.A., Lauretta, D.S., the OSIRISREx Team, 2019. Shape of (101955) Bennu indicative of a rubble pile with internal stiffness. Nat. Geosci. 12, 247-252. doi.org/10.1038/s41561-019-0330-x.

Barnouin, O.S., Daly, M.G., Palmer, E.E., Johnson, C.L., Gaskell, R.W., Al Asad, M., Bierhaus, E.B., Craft, K.L., Ernst, C.M., Espiritu, R.C., Nair, H., Neumann, G.A., Nguyen, L., Nolan, M.C., Mazarico, E.M., Perry, M.E., Philpott, L.C., Roberts, J.H., Steele, R.J., Seabrook, J., Susorney, H.C.M., Weirich, J.R., Lauretta, D.S., 2020. Digital Terrain Mapping by the OSIRIS-REx mission. Planet. Space. Sci. 180, 104764. doi.org/10.1016/j.pss.2019.104764.

Becker K.J., Berry, K.L., Mapel, J.A., Walldren, J.C., 2017. A New Approach to Create Image Control Networks in ISIS. $3^{\text {rd }}$ Planetary Data Workshop and the Planetary Mappers Annual Meeting, 12-15 June, Flagstaff, AZ, Abstract 7133.

Becker, K.J., Becker, T.L., Edmundson, K.L., Golish, D.R., Bennett, C.A., Porter, N.A., DellaGiustina, D.N., Daly, M.G., Palmer, E., Weirich, J., Barnouin, O.S., Philpott, L., Al Asad, M.M., Seabrook, J.A., Johnson, C.L., Rizk, B., Lauretta, D.S., 2020. Photogrammetric Control of Candidate Sample Sites on (101955) Bennu. Lunar Planet Sci., LI, Abstract 2569.

Bennett, C.A., DellaGiustina D.N., Becker, K.J., Becker T.L., Edmundson, K.L., Golish, D. R, Bennett, R. J., Burke, K.N., Cue, C.N.U., Clark, B.E., Contreras, J., Deshapriya, J.D.P., Drouet d'Aubigny, C., Jawin, E.R., Nolan, T.Q., Porter, N.A., Riehl, M.M., Roper, H.L., Rizk, B., Tang, Y., Zeszut, Z., Gaskell, R.W., Palmer E.E., Weirich, J.R., Al Asad, M.M., Philpott, L., Daly, M.G., Barnouin, O.S., Enos, H.L., Lauretta, D.S., 2020. A HighResolution Global Basemap of (101955) Bennu. Icarus. doi.org/10.1016/j.icarus.2020.113690.

Bierhaus, E.B., Clark, B.C., Harris, J.W., Payne, K.S., Dubisher, R.D., Wurts, D.W., Hund, R.A., Kuhns, R.M., Linn, T.M., Wood, J.L., May, A.J., Dworkin, J.P., Beshore, E., Lauretta, D.S., the OSIRIS-REx Team, 2018. The OSIRIS-REx Spacecraft and the Touch-and-Go Sample Acquisition Mechanism (TAGSAM). Space Sci. Rev., 214, 107. doi.org/10.1007/s11214-018-0521-6.

Brown, D.C., 1966. Decentering Distortion of Lenses, Photogrammetric Engineering, 32(3), 444-462.

Christensen, P.R., Anwar, S., Burris, M.E., Carter, S.R., Dickenshied, S.L., Noss, D.D., Hagee, W.R., Rios, K.J., Wren, P.F., 2018. J-Asteroid: JMARS for Asteroids and Other Small Bodies. Lunar Planet Sci., XLIX, Abstract 2788.

Daly, M.G., Barnouin, O.S., Dickinson, C., Seabrook, J., Johnson, C.L., Cunningham, G., Haltigin, T., Gadreau, D., Brunet, C., Aslam, I., Taylor, A., Bierhaus, E.B., Boynton, W., Nolan, M., Lauretta, D.S., 2017. The OSIRIS-REx Laser Altimeter (OLA) Investigation and Instrument. Space Sci. Rev., 212, 899-924. doi.org/10.1007/s11214-017-0375-3.

DellaGiustina, D.N., Bennett C.A., Becker, K., Golish, D.R., Le Corre, L., Cook, D.A., Edmundson, K.L., Chojnacki, M., Sutton, S.S., Milazzo, M.P., Carcich, B., Nolan, M.C., Habib, N., Burke, K.N., Becker, T., Smith, P.H., Walsh, K.J., Getzandanner, K.,
Wibben, D.R., Leonard, J.M., Westermann, M.M., Polit, A.T., Kidd Jr, J.N., Hergenrother, C.W., Boynton, W.V., Backer, J., Sides, S., Mapel, J., Berry, K., Roper, H., Drouet d'Aubigny, C., Rizk, B., Crombie, M.K., Kinney-Spano, E.K., de León, J., Rizos, J.L., Licandero, J., Campins, H.C., Clark, B.E., Enos, H.L., Lauretta, D.S., 2018. Overcoming the Challenges Associated with Image-Based Mapping of Small Bodies in Preparation for the OSIRIS-REx Mission to (101955) Bennu. Earth Space Sci., 5, 929-949. doi.org/10.1029/2018EA000382.

DellaGiustina, D.N., Emery, J.P., Golish, D.R., Rozitis, B., Bennett, C.A., Burke, K.N., Ballouz, R.-L., Becker, K.J., Christensen, P.R., Drouet d'Aubigny, C.Y., Hamilton, V.E., Reuter, D.C., Rizk, B., Simon, A.A., Asphaug, E., Bandfield, J.L., Barnouin, O.S., Barucci, M.A., Bierhaus, E.B., Binzel, R.B., Bottke, W.F., Bowles, N.E., Campins, H., Clark, B.C., Clark, B.E., Connolly Jr., H.C., Daly, M.G., de Leon, J., Delbo', M., Deshapriya, J.D.P., Elder, C.M., Fornasier, S., Hergenrother, C.W., Howell, E.S., Jawin, E.R., Kaplan, H.H., Kareta, T.R., Le Corre, L., Li, J.-Y., Licandro, J., Lim, L.F., Michel, P., Molaro, J., Nolan, M.C., Pajola, M., Popescu, M., Rizos Garcia, J.L., Ryan, A., Schwartz, S.R., Shultz, N., Siegler, M.A., Smith, P.H., Tatsumi, E., Thomas, C.A., Walsh, K.J., Wolner, C.W.V., Zou, X.-D., Lauretta, D.S., the OSIRIS-REx Team, 2019. Properties of rubble-pile asteroid (101955) Bennu from OSIRIS-REx imaging and thermal analysis. Nat. Astron., 3, 341-351. doi.org/10.1038/s41550-019-0731-1.

Edmundson, K.L., Cook, D.A., Thomas, O.H., Archinal, B.A., Kirk, R.L., 2012. Jigsaw: The ISIS3 Bundle Adjustment for Extraterrestrial Photogrammetry. ISPRS Ann. Photogramm. Remote Sens. Spatial Inf. Sci., I-4, 203-208. doi.org/10.5194/isprsannals-I-4-203-2012.

Gaskell, R.W., Barnouin-JHA, O.S., Scheeres, D.J., Konopliv, A.S., Mukai, T., Abe, S., Saito, J., Ishiguro, M., Kubota, T., Hashimoto, T., Kawaguchi, J., Yoshikawa, M., Shirakawa, K., Kominato, T., Hirata, N., Demura, H., 2008. Characterizing and navigating small bodies with imaging data. Meteorit. Planet. Sci. 43(6), 1049-1061. doi.org/10.1111/j.1945-5100.2008.tb00692.x.

Golish D.R., DellaGiustina, D.N., Clark, B.E., Bennett, C.A., Li, J.-Y., Zou, X.D., Lauretta, D.S., 2017. Photometric Modeling of Simulated Surface-Resolved Bennu Images. $3^{\text {rd }}$ Planetary Data Workshop and the Planetary Mappers Annual Meeting, 12-15 June, Flagstaff, AZ, Abstract 7069.

Golish, D.R., Drouet d'Aubigny, C., Rizk, B., DellaGiustina, D.N., Smith, P.H., Becker, K., Shultz, N., Stone, T., Barker, M.K., Mazarico, E., Tatsumi, E., Gaskell, R.W., Harrison, L., Merrill, C., Fellows, C., Williams, B., O’Dougherty, S., Whiteley, M., Hancock, J., Clark, B.E., Hergenrother, C.W., Lauretta, D.S., 2020a. Ground and In-Flight Calibration of the OSIRIS-REx Camera Suite. Space Sci. Rev. 216, 12. doi.org/10.1007/s1 1214-019-0626-6.

Golish, D.R., DellaGiustina, D.N., Li, J.-Y., Clark, B.E., Zou, X.D., Smith, P.H., Rizos, J.L., Hasselmann, P.H., Bennett, C.A., Fornasier, S., Ballouz, R.-L., Drouet d'Aubigny, C., Rizk, B., Daly, M.G., Barnouin, O.S., Philpott, L., Al Asad, M.M., Seabrook, J.A., Johnson, C.L., Lauretta, D.S., 2020b. Diskresolved Photometric Modelling and Properties of Asteroid (101955) Bennu. Icarus. doi.org/10.1016/j.icarus.2020.113724.

Lauretta, D.S., Balram-Knutson, S.S., Beshore, E., Boynton, W.V., Drouet d'Aubigny, C., DellaGiustina, D.N., Enos, H.L., Golish, D.R., Hergenrother, C.W., Howell, E.S., Bennett, C.A., 
Morton, E.T., Nolan, M.C., Rizk, B., Roper, H.L., Bartels, A.E.,Bos, B.J., Dworkin, J.P., Highsmith, D.E., Lorenz, D.A., Lim, L.F., Mink, R., Moreau, M.C., Nuth, J.A., Reuter, D.C., Simon, A.A., Bierhaus, E.B., Bryan, B.H., Ballouz, R., Barnouin, O.S., Binzel, R.P., Bottke, W.F., Hamilton, V.E., Walsh, K.J., Chesley, S.R., Christensen, P.R., Clark, B.E., Connolly, H.C., Crombie, M.K., Daly, M.G., Emery, J.P., McCoy, T.J., McMahon, J.W., Scheeres, D.J., Messenger, S., NakamuraMessenger, K., Righter, K., Sandford, S.A., 2017. OSIRIS-REx: Sample Return from Asteroid (101955) Bennu. Space Sci. Rev. 212, 925-984. doi.org/10.1007/s11214-017-0405-1.

Lauretta, D.S., DellaGiustina, D.N., Bennett, C.A., Golish, D.R., Becker, K.J., Balram-Knutson, S.S., Barnouin, O.S., Becker, T.L., Bottke, W.F., Boynton, W.V., Campins, H., Clark, B.E., Connolly Jr, H.C., Drouet d'Aubigny, C.Y., Dworkin, J.P., Emery, J.P., Enos, H.L., Hamilton, V.E., Hergenrother, C.W., Howell, E.S., Izawa, M.R.M., Kaplan, H.H., Nolan, M.C., Rizk, B., Roper, H.L., Scheeres, D.J., Smith, P.H., Walsh, K.J., Wolner, C.W.V., The OSIRIS-REx Team, 2019. The unexpected surface of asteroid (101955) Bennu. Nature, 568, 55-60. doi.org/10.1038/s41586-019-1033-6.

Pelgrift, J.Y., Sahr, E.M., Nelson, D.S., Jackman, C.D., Benhacine, L., Bos, B.J., Rizk, B., d'Aubigny, C., Golish, D., Dellagiustina, D., Lauretta, D.S., 2018. In-flight calibration of the OSIRIS-REx optical navigation imagers. Paper presented at $1 s t$ Annual RPI Workshop on Image-Based Modeling and Navigation for Space Applications, Rensselaer Polytechnic Institute, Troy, NY, June 4-5.

Polit, A.T., Enos, H. L., Boynton, W.V., Lambert, D., Westermann, M.M., Kidd, J.N., Garcia, R., Becker, T., BalramKnutson, S.S., Harshman, K., Lauretta, D.S., 2020. Science Operations Planning for the OSIRIS-REx Asteroid Sample Return Mission. Lunar Planet Sci., LI, Abstract 2430.

Rizk, B., Drouet d'Aubigny, C., Golish, D., Fellows, C., Merrill, C., Smith, P., Walker, M.S., Hendershot, J.E., Hancock, J., Bailey, S.H., DellaGiustina, D.N., Lauretta, D.S., Tanner, R., Williams, M., Harshman, K., Fitzgibbon, M., Verts, W., Chen, J., Connors, T., Hamara, D., Dowd, A., Lowman, A., Dubin, M., Burt, R., Whiteley, M., Watson, M., McMahon, T., Ward, M., Booher, D., Read, M., Williams, B., Hunten, M., Little, E., Saltzman, T., Alfred, D., O’Dougherty, S., Walthall, M., Kenagy, K., Peterson, S., Crowther, B., Perry, M.L., See, C., Selznick, S., Sauve, C., Beiser, M., Black, W., Pfisterer, R.N., Lancaster, A., Oliver, S., Oquest, C., Crowley, D., Morgan, C., Castle, C., Doninguez, R., Sullivan, M., 2018. OCAMS: The OSIRIS-REx Camera Suite. Space Sci. Rev., 214, 26. doi.org/10.1007/s11214017-0460-7.

Sides, S.C., Becker, T.L., Becker, K.J., Edmundson, K.L., Backer, J.W., Wilson, T.J., Weller, L.A., Humphrey, I.R., Berry, K.L., Shepherd, M.R., Hahn, M.A., Rose, C.C., Rodriguez, K., Paquette, A.C., Mapel, J.A., Shinaman, J.R., Richie, J.O., 2017. The USGS Integrated Software for Imagers and Spectrometers (ISIS 3) Instrument Support, New Capabilities, and Releases. Lunar Planet Sci., XLVIII, Abstract 2739. 